\title{
Accuracy of Ultrasound and Magnetic Resonance Imaging (MRI) in Penile Fracture Mapping for Modified Surgical Repair
}

\author{
Mohammad Zare Mehrjardi, ${ }^{1, *}$ Mohsen Darabi, ${ }^{2}$ Seyed Morteza Bagheri, ${ }^{2}$ and Shaghayegh Sadat
}

\author{
Khabbaz \\ ${ }^{1}$ Department of Radiology, Shahid Beheshti University of Medical Sciences (SBMU) \\ ${ }^{2}$ Department of Radiology, Iran University of Medical Sciences, Tehran, Iran \\ ${ }^{3}$ Department of Radiology, ShahidBeheshti University of Medical Sciences (SBMU) \\ "Corresponding author: Mohammad Zare Mehrjardi, Department of Radiology, Shahid Beheshti University of Medical Sciences (SBMU). E-mail: E-mail: zare@sbmu.ac.ir
}

Received 2016 December 21; Accepted 2017 February 08.

\begin{abstract}
Background: Penile fracture is a relatively rare urologic emergency. It is defined as traumatic rupture in tunica albuginea of corpus cavernosum. Traditionally, surgeon explores the penis in full-length by complete degloving of its skin and repairs any defect in the tunica. Imaging modalities such as ultrasound and MRI may be of help to reveal the exact site of tunica defect preoperatively for tailoring the surgical repair by making a direct incision over the defect. Tunica albuginea is a hyperechoic layer in ultrasound covering corpora cavernosa, and its tear appears as a hypoechoic defect in this band. In MRI, tunica is seen as a low-signal intensity layer in all conventional pulse sequences around the corpora cavernosa, and its tear is evident as a discontinuity in this layer. In addition, imaging modalities may demonstrate associated injuries such as hematoma, urethral rupture, and corpus spongiosum injury.

Objectives: To investigate accuracy of ultrasound and magnetic resonance imaging (MRI) in penile fracture diagnosis and preoperative mapping.

Methods: 18 consecutive patients included in the study during two years prospectively. Ultrasound and MRI were performed in all patients and interpreted by two expert radiologists independently. The defect site was mapped on a designed platform preoperatively using each modality blinded to the result of other modality. All patients were explored surgically by an expert surgeon using complete degloving of the penis technique, which was the routine procedure in our tertiary referral center. The surgeon was blinded to the radiologic mapping, and the surgical results were considered as the gold-standard. Detection rate, and agreement between preoperative imaging mapping and surgical result were determined for each modality.

Results: Mean age of patients was $28.2 \pm 7.3$ years-old. Most penile fractures were occurred during sexual intercourse (89\%). Most common location of tunica rupture was mid-shaft of penis (67\%), and mean length of tunica defect was $14.8 \pm 3.2 \mathrm{~mm}$. All patients had associated hematoma, but no one revealed urethral injury. Detection rate of ultrasound and MRI was $89 \%$ and $100 \%$, respectively. Ultrasound was unable to detect tunica rupture in 2 patients (11\%), all of which revealed tears at the penile base in MRI and during surgical exploration. Ultrasound mapped tear location correctly in 13 patients (kappa, $0.26 ; \mathrm{P}=0.045$ ), while MRI mapped it precisely in 17 cases (kappa, $0.89 ; \mathrm{P}=1.00$ ).

Conclusions: Both imaging modalities may be used for detecting tunica tear especially in atypical cases that physical examination is equivocal. However, MRI is more accurate in preoperative mapping of rupture location for performing a modified less invasive surgery.
\end{abstract}

This is an abstract presented in the 33rd Iranian congress of radiology (ICR) and the 15th congress of Iranian radiographic science association (IRSA) 